\title{
Selective Cortical Alteration after Hypoxic-Ischemic Injury in the Very Immature Rat Brain
}

\author{
STÉPHANE V. SIZONENKO, ERNEST SIRIMANNE, YVETTE MAYALL, PETER D. GLUCKMAN, \\ TERRIE INDER, AND CHRIS WILLIAMS \\ Unité de Développement, Department of Paediatrics, Hopital des Enfants, 1211 Geneva 14, Switzerland \\ [S.V.S.]; The Liggins Institute, School of Medicine, University of Auckland, 92019 Auckland, New Zealand \\ [S.V.S., E.S., Y.M., P.D.G., C.W.]; Murdoch and Childrens Research Institute, Howard Florey Institute, \\ Royal Women's Hospital, 3053 Melbourne, Australia [T.I.]
}

\begin{abstract}
Distinctive cerebral lesions with disruptions to the developing white matter are found in very low birth weight (VLBW) infants. Although hypoxia-ischemia (HI) is a causal pathway, the pathogenesis of cerebral white matter injury in the VLBW infant is not fully understood. Pertinent murine models would facilitate the investigation of the processes leading to these cerebral lesions and enable the evaluation of therapeutic strategies. Postnatal d 3 (P3) rats are at a stage of cortical oligodendroglial maturation and axonal outgrowth similar to very preterm infants. Our aim was to characterize the effects of a focal hypoxic-ischemic injury at P3 on subsequent cerebral development. Three groups of P3 Wistar rats were investigated: group I underwent right carotid ligation followed by $6 \%$ hypoxia for $30 \mathrm{~min}(\mathrm{HI})$, group 2 had carotid ligation only, and group 3 had no intervention. At P21, in the $\mathrm{HI}$ group, the right cortical area was reduced compared with controls $(p<0.01)$. There were no significant alterations in the size of the dorsal hippocampus, striatum, and thalamus. The cortical myelinated area was reduced in the $\mathrm{HI}$ animals compared
\end{abstract}

\section{ABSTRACT}

with controls $(p<0.01)$. There was a corresponding loss of myelinated axons extending up into the cortex, with deep cortical neuronal and axonal architecture markedly disrupted. Glial fibrillary acidic protein immunohistology showed a reactive gliosis in the deep parietal cortex $(p<0.01)$. Moderate HI injury in the immature rat brain compromised cortical growth and led to a selective alteration of cortical myelinated axons with persistent gliosis. These alterations induced at P3 by unilateral HI share neuropathological similarities with the diffuse white matter lesions found in VLBW infants. (Pediatr Res 54: 263-269, 2003)
HI, hypoxia-ischemia
Abbreviations
WM, white matter
PVL, periventricular leukomalacia
MBP, myelin basic protein
GFAP, glial fibrillary acidic protein

Of those infants born weighing $<1500 \mathrm{~g}, 25-50 \%$ will later exhibit developmental disabilities and up to $5-15 \%$ will display a more major cerebral palsy $(1,2)$. The pattern of brain damage associated with prematurity is unique, with a specific loss of the developing cerebral WM (3), and differs markedly from the predominantly gray matter injuries that occur in the more mature CNS. The distinct cystic WM lesions termed PVL and a more subtle and diffuse lesion with loss of WM volume and ventriculomegaly $(3,4)$ feature with subsequent abnormal

Received May 30, 2002; accepted December 4, 2002.

Correspondence: Stéphane Sizonenko, Unité de Développement, Département de Pédiatrie, Hopital des Enfants, 6 rue Willy Donzé, 1211 Geneva 14 Switzerland; e-mail: stephane.sizonenko@medecine.unige.ch

Supported by grants from the Health Research Council of New Zealand and from New Zealand Lotteries Board. S.V.S. was recipient of postdoctoral fellowships from the Eugenio Litta Foundation and the Geneva University Hospital (Switzerland).

DOI: 10.1203/01.PDR.0000072517.01207.87 development of cortical gray matter and alterations in the central and distal WM microstructure (5-7). The etiology of these injuries in the premature infant is not fully understood (8), although HI injury, inflammation associated with cytotoxic cytokines, and an increased vulnerability of oligodendrocytes precursors to HI appear to be important $(9,10)$. Prevention of these adverse outcomes requires an understanding of the pathogenesis of the lesion and the subsequent alterations in brain development.

It is difficult to precisely compare neural maturation between species. However, the cortex of the 12- to 14-d-old rat corresponds approximately to that of a term human newborn with myelination of the fiber tracts beginning on approximately d 11 in the rat. The 7-d-old rat is widely used and generally considered to reflect the maturity of a 30- to 34-wk gestational human fetus. Neurogenesis is largely complete by birth in the rat, suggesting that $\mathrm{d} 1$ in the rat corresponds to about $20 \mathrm{wk}$ 
gestation in human $(11,12)$. The staging of cerebral axonal outgrowth at $3 \mathrm{~d}$ in the rat (13) is equivalent to very preterm infants (14). In addition, recent results show that markers of cortical oligodendroglial progenitor maturation suggest the 3 -d-old rat is equivalent to the very premature infants (24-28 wk) (15). Together, these data suggest that the 3-d-old rat is comparable, in terms of cortical and WM development, to the very preterm infant aged about $24-28$ wk of gestational age.

To be able to address the issues of diffuse WM injuries and subsequent altered brain development of the very preterm infant, we have adapted the model of moderate focal $\mathrm{HI}$ injury in the postnatal d $21(\mathrm{P} 21)$ rat (16) to the immature P3 rat and characterized the effect of a moderate unilateral $\mathrm{HI}$ injury on subsequent cerebral growth, extent of myelination, and gliosis.

\section{MATERIALS AND METHODS}

The University of Auckland Ethics Committee approved these studies. Three-day-old Wistar rats $(58-80 \mathrm{~h}$ ) from three litters (38 animals), weighing between 8 and $10 \mathrm{~g}$ were randomly allocated to three different study groups: HI group, $n=25$; carotid ligation only (L) group, $n=8$; and the control (N) group, $n=5$. Unilateral common carotid artery occlusion combined with hypoxia was used to produce $\mathrm{HI}$ injury in these $\mathrm{P} 3$ rats. The animals were anesthetized with halothane (5\% induction, 3\% maintenance), during which the right common carotid artery was coagulated using a pair of fine electrosurgical forceps under an operating microscope. Each animal was placed on a warmed heating pad until they had recovered from the effects of the anesthetic. Once all the animals had fully recovered, they were placed in an infant incubator at $37^{\circ} \mathrm{C}$ in $85-90 \%$ humidity for $30 \mathrm{~min}$ before undergoing $6 \% \mathrm{O}_{2}$ hypoxia for $30 \mathrm{~min}$ in the same thermoneutral environment. The animals were returned to the dam until they reached $21 \mathrm{~d}$ of age, whereupon they were killed by an intraperitoneal overdose of sodium pentobarbitone and the brains were perfused with saline followed by $4 \%$ paraformaldehyde and cacodylic acid. The brains were removed and immersed in the same fixative for $48 \mathrm{~h}$. They were then sectioned into $3 \times 2 \mathrm{~mm}$ coronal sections using a coronal brain matrix (RBM-3000C, ASI Instruments, Houston, TX, U.S.A.) and dehydrated overnight through a series of alcohols, cleared in chloroform, and finally embedded in paraffin.

Histology. Paraffin sections, $4 \mu \mathrm{m}$ thick, were cut using a rotary Leica microtome, collected on chrom-aluminum gelatin coated slides, dewaxed in xylene, passed through graded alcohols, and brought to water. Adjacent sections were stained using thionin and acid fuchsin, dehydrated, and mounted using Micromount (Surgipath, Richmond, IL, U.S.A.) or used for immunohistology.

MBP and GFAP. Paraffin sections, $4 \mu \mathrm{m}$ thick, were collected to chrome aluminum coated slides, dewaxed, and brought to water through graded alcohols. Slides were then placed in 0.1M PBS containing $0.05 \%$ Tween 20 (PBS-T) for $5 \mathrm{~min}$. Endogenous peroxidase was blocked for $30 \mathrm{~min}$ in $1 \% \mathrm{H}_{2} \mathrm{O}_{2}$ in methanol. Nonspecific protein binding was blocked with $2.5 \%$ normal goat serum in $0.5 \%$ BSA-PBS-T for $30 \mathrm{~min}$. The sections were incubated with a primary monoclonal mouse antibody for MBP (MAb 5274, 1:500, Roche Molecular Biochemicals, Mannheim, Ger- many) or a primary polyclonal rabbit antibody for GFAP (Z334, 1:400, DAKO, Glostrup, Denmark) diluted in in PBS-T overnight at $4^{\circ} \mathrm{C}$. This was followed with an overnight incubation with biotinylated goat anti-mouse IgG and anti-rabbit IgG (Amersham Pharmacia Biotech, Piscataway, NJ, U.S.A.), respectively, using a 1:200 dilution at $4^{\circ} \mathrm{C}$. The slides were then incubated for $3 \mathrm{~h}$ at room temperature with streptavidin biotinylated horseradish peroxidase (Amersham Pharmacia Biotech) and developed with nickel-enhanced diaminobenzidine (Sigma Chemical, St. Louis, MO, U.S.A.). Stained sections were mounted using Micromount (Surgipath). In every experiment, a section from a normal uninjured P21 rat was used as a positive control and a specimen incubated with PBS and normal serum in place of the secondary antibody to the primary antibody was used as a negative control.

Analysis. Using SigmaScan Image analysis software (Jandel Scientific, San Rafael, CA, U.S.A.), under a microscope (Esselte Leitz, Stuttgart, Germany) the cortical area between the rhinal sulcus, the cingulum (Fig. 1A) and the external boundaries of the corpus callosum as well as the area of positive MBP immunostaining between the rhinal sulcus and the cingulum (Fig. 1B) were measured. These measures were separately carried out on both hemispheres and on three separate sections at the level of the striatum, the dorsal hippocampus, and the ventral hippocampus. The area of gray matter in the cortex was calculated, where the area of MBP positive cortical WM was subtracted from the overall cortical area. Measurements were taken of the striatum (Fig. 1C) in the anterior
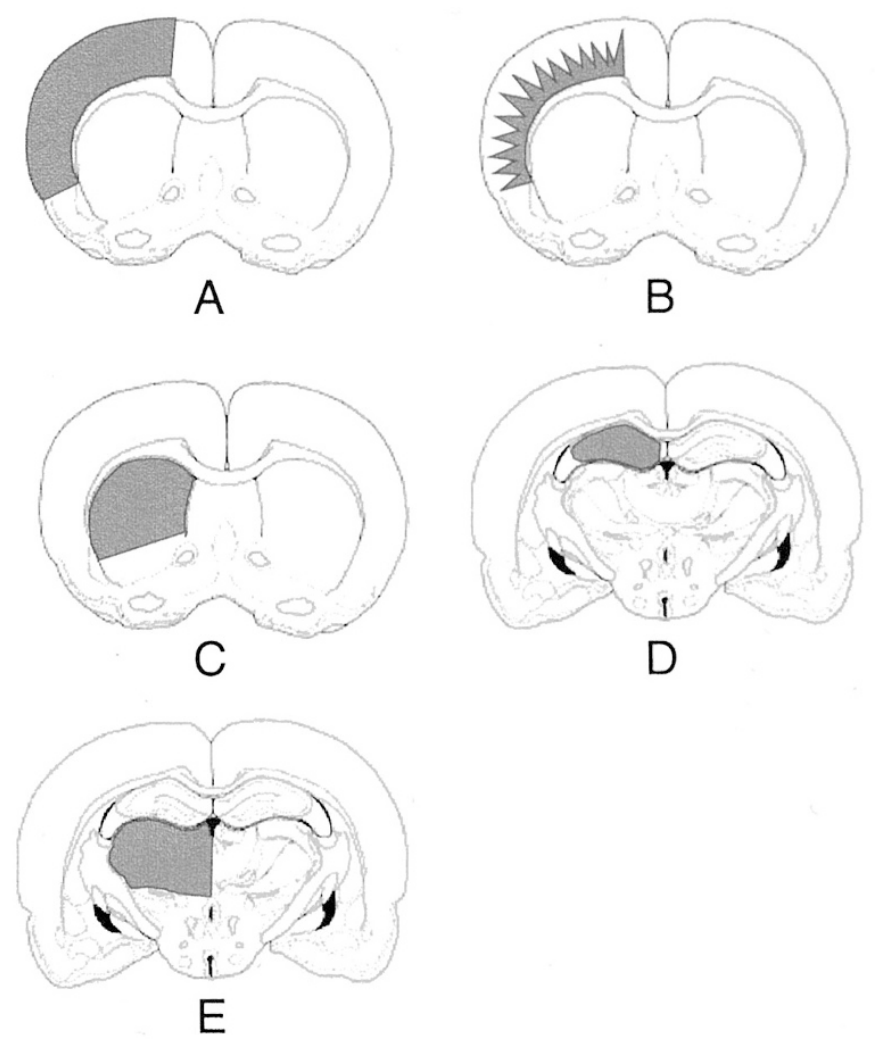

Figure 1. Neuroanatomical assessment. (A) Cortex, $(B)$ cortical WM, $(C)$ striatum, $(D)$ dorsal hippocampus, and $(E)$ thalamus. The shaded areas represent the neuroanatomical regions assessed by image analysis for alterations in size. 
section and of the hippocampus (Fig. 1D), thalamus (Fig. 1E) and corpus callosum. No difference in area between the injured animals and the controls was observed in these four latter regions, therefore, they were not investigated any further. In the parietal cortex, as delimited above, GFAP immunostaining was assessed by scoring the level of positive cells in both sides: $0=$ rare GFAP-positive cells, $1=$ moderate density of GFAPpositive cells, and $2=$ high density of GFAP-positive cells.

Data analysis. To avoid any variability resulting from tissue handling and staining, the absolute areas were expressed as the right to left interhemispheric ratio of the measured areas. A mixed ANOVA model was used to compare regions and the three different study groups. Interactions between treatment and region were included in the model. Posthoc comparisons of cortical area and cortical WM were made with Scheffe's test. The left-to-right amount of GFAP-positive cells in the parietal cortex was compared using a Wilcoxon signed rank test. Significance was defined as $p<0.05$. Results are expressed as mean \pm SEM.

\section{RESULTS}

Of the 25 animals in the HI group, two died during the hypoxia (mortality, $8.6 \%$ ). No animals died in the ligated or control groups.

Size of cerebral structures. The right-to-left (R/L) ratio of the parietal cortex was reduced after the HI injury when compared with the normal group (R/L-HI: $0.88 \pm 0.14 ; \mathrm{R} / \mathrm{L}-\mathrm{N}$ :
$0.96 \pm 0.1 ; p<0.01$ ) (Fig. 2A). This lesion was apparent as a reduction in the size in the region from the rhinal sulcus up to the cingulum. The $\mathrm{R} / \mathrm{L}$ ratio of the cortical area of the HI group was also reduced compared with the ratio of corresponding region in the ligated only control group (R/L-HI: $0.88 \pm 0.14$; R/L-L: $0.99 \pm 0.1 ; p<0.01$ ) (Fig. $2 A$ ). Within the HI group there were no significant differences in the reduction of cortical area between the three sections (anterior, parietal, and posterior) analyzed. Twenty-one of the 23 injured brains (91\%) had evidence of ipsilateral histopathological changes when examined using both thionin-acid fuchsin and MBP staining. In addition, this injury did not significantly alter the area of the striatum, thalamus, or dorsal hippocampus (Fig. 2, $B-D$ ). Further no overt pathologic changes within the thalamus, dorsal hippocampus, or striatum were seen using both thioninacid fuchsin and MBP staining.

Extent of cortical myelination. MBP immunostaining was present in the corpus callosum (CC), with areas of positive staining extending into the cortical tissue above the CC. Within the ipsilateral parietal cortex, there was clear loss of the MBP-positive intracortical WM in the HI group when compared with the $\mathrm{N}$ group (R/L-HI: $0.88 \pm 0.15$; R/L-N: $1.0 \pm$ $0.1 ; p<0.01)$ and to the ligated only group $(\mathrm{R} / \mathrm{L}-\mathrm{L}: 1.0 \pm 0.1$; $p<0.01$ ) (Fig. 2E). In contrast, there was no significant change in the area of gray matter when compared with the contralateral hemisphere. Again, no significant difference in
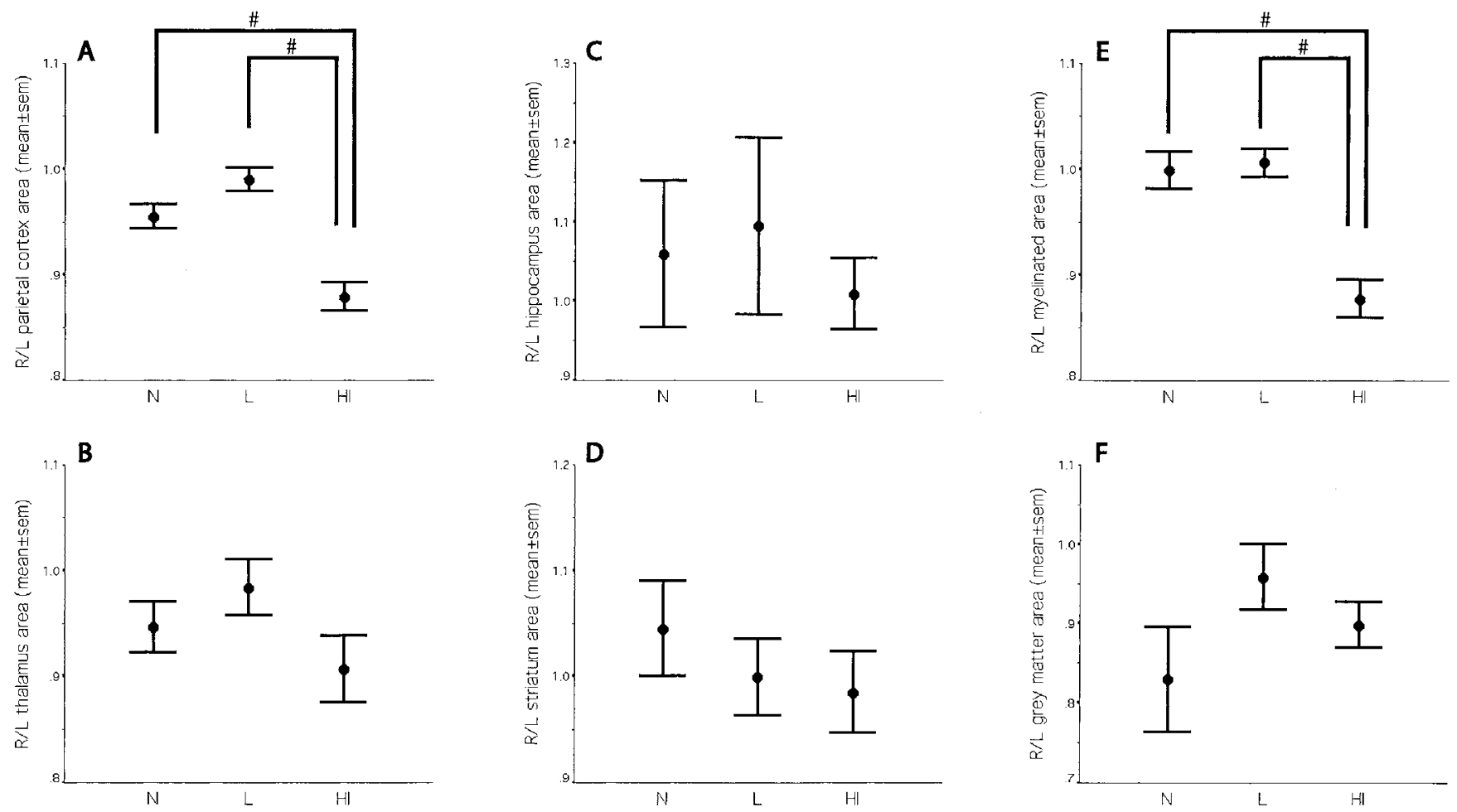

Figure 2. Effect of HI on different regions of the brain and on cortical WM and gray matter. Right-to-left (R/L) ratio of the area of different regions of the normal, ligated only, and HI animals. (A) The R/L area of parietal cortex in the HI brains (HI) was reduced compared with the normal (N) and ligated only (L) groups $\left({ }^{\#} p<0.01\right)$. (B) There was not a significant difference between the $\mathrm{R} / \mathrm{L}$ area of the thalamus after $\mathrm{HI}$ compared with $\mathrm{N}$ and $\mathrm{L}$ groups. $(C)$ There was not a significant difference between the $\mathrm{R} / \mathrm{L}$ area of the hippocampus after $\mathrm{HI}$ compared with $\mathrm{N}$ and $\mathrm{L}$ groups. $(D)$ Similarly, the size of striatum was not altered. $(E)$ The $\mathrm{R} / \mathrm{L}$ area of MBP immunostaining within the parietal cortex in the HI brains was reduced in the ipsilateral hemisphere compared with the $\mathrm{N}$ and $\mathrm{L}$ animals $\left({ }^{\#} p<0.01\right)$. $(F)$ There was no significant difference between groups in the $\mathrm{R} / \mathrm{L}$ area of gray matter in the cortex. 
the cortical WM area between the anterior and posterior levels of the brain in the HI group was seen. In the CC, no significant difference in the $\mathrm{R} / \mathrm{L}$ ratio between the $\mathrm{N}, \mathrm{L}$, and $\mathrm{HI}$ groups of the area of positive MBP staining was found.

Thionin-acid fuchsin staining. After an $\mathrm{HI}$ challenge at $\mathrm{P} 3$, no cystic lesions were seen at P21. The upper layers of cortical neurons appeared to be intact. The histopathological changes were predominantly manifest as a conical loss of cells that occurred in the ipsilateral deep parietal cortical regions, occasionally projecting up to layer IV (Fig. 3, $A$ and $B$ ). Scattered acidophilic (dead/dying) neurons were seen in these areas of conical loss in conjunction with an infiltration of small, dark-stained microgliallike cells or astrocytes (Fig. 3, $C$ and $D$ ). In the deep ipsilateral cortex, a disorganization of the neuroarchitectural pattern could be seen when compared with the contralateral hemisphere (Fig. 3, $C$ and $D$ ). Microscopic evaluation revealed that 53\% (12/23) posterior brain sections showed the overt histopathological changes described above. Of the tissue slices drawn from the midbrain, $43 \%(10 / 23)$ and $26 \%(6 / 23)$ in the anterior sections showed these histologic changes. No modifications were seen in the striatum, thalamus, or hippocampus.

MBP immunostaining. Clear changes in the pattern of MBP-positive fibers could be seen (Fig. 4). The change that was most apparent was the rearrangement of the distribution of the fibers resulting in compacting or clumping of myelinated fibers. Changes to the pattern of MBP staining were evident in $78 \%$ of the brains (18/23 brains). Of the 18 brains, only five (28\%) showed a mild decrease in the staining density of the ipsilateral hemisphere. In the remain- ing brains $(72 \%)$, clear columnar patches marked by the absence of myelinated fibers side by side with clumped myelinated fibers (Fig. 4, $A$ and $B$ ) were seen. These lesions occurred predominantly in the parietal cortex. A different pattern was seen in the posterior occipital cortex, where there appeared to be a distinct loss of volume with less of the structural changes (Fig. $4 C$ ).

GFAP immunostaining. GFAP-positive astrocytes were seen at the age of P21 in the ipsilateral parietal cortex: 21 brains out of $24(87 \%)$ had an increased score of GFAP-positive cells in the ipsilateral cortex $(\mathrm{RH})$ compared with the contralateral $(\mathrm{LH})$ (HI-RH: $1.08 \pm 0.13$; HI-LH: $0.21 \pm 0.01 ; p<0.01$ ). Very few GFAP-positive astrocytes could be seen in the contralateral parietal cortex (Fig. 5A). In the mildly damaged brains, the cellular distribution of GFAP staining was diffuse within the cortical layers IV-VI (Fig. 5B). In more severely injured animals, there was a denser columnar pattern with gliotic scars superimposed on the diffuse reaction within layers IV-VI (Fig. 5C). In these columns, the astrocytes showed larger cell body and thicker processes (Fig. 5D). In five brains with the most severe ipsilateral injury, increased scattered GFAP-positive cells were present in the contralateral cortex. In the corpus callosum, external capsule and internal capsule, there were a high number of activated astrocytes on both sides of the brain in all animals.

\section{DISCUSSION}

The results indicate a specific reduction in the size at P21 of the parietal cortex and of the cortical WM after a moderate HI
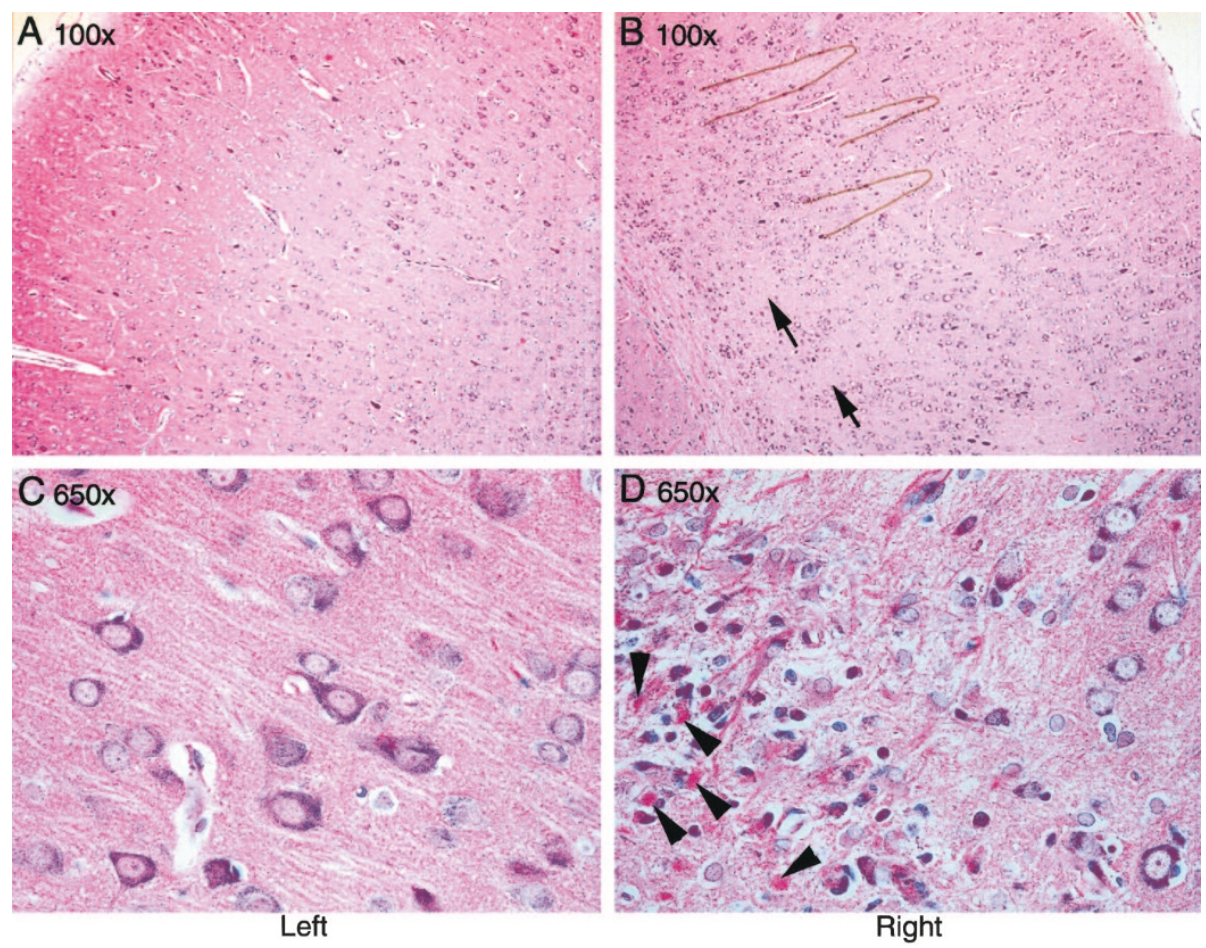

Figure 3. Effect of $\mathrm{HI}$ on neuronal loss and on the neuroarchitectural pattern. Example of the patchy conical loss of cortical neurons (outlined in $B$ ) in the right parietal cortex extending up to layer IV-V compared with the noninjured left parietal cortex $(A)$, and a loss of neurones is also noted in the deeper cortical layers (arrows, B). At higher magnification, the presence of pink acidophilic cells can be noted (D, arrowheads) with an important infiltration of microglial cells or astrocytes. Note also the alteration of the regular neuroarchitectural pattern when compared with the left uninjured side $(C)$. Stain: thionin-acid fuchsin; magnification: $A, B=100 \times ; C, D=650 \times$. 

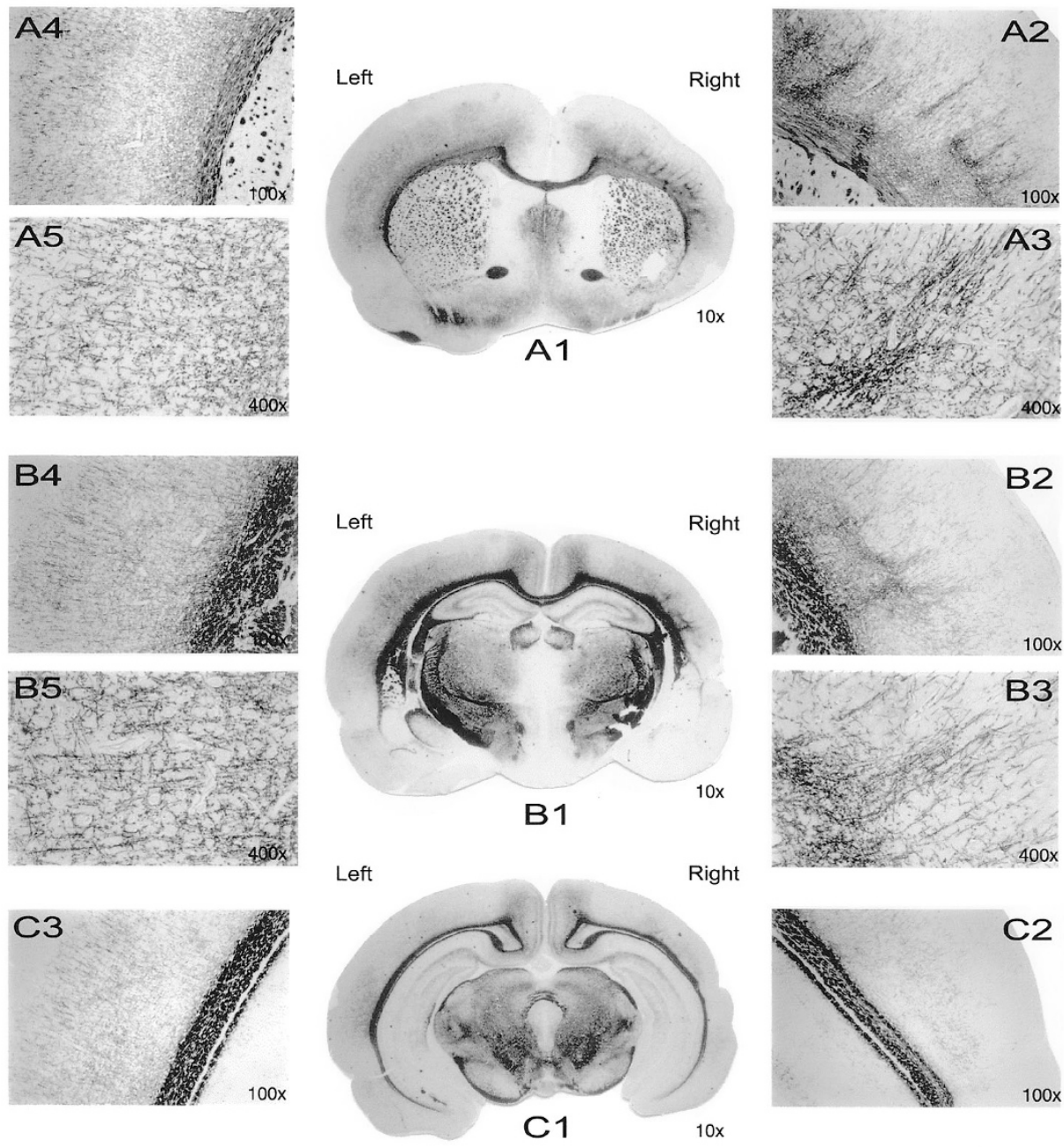

Figure 4. Effect of $\mathrm{HI}$ on the pattern of myelin. MBP immunostaining of the brain at $\mathrm{P} 21$ after $\mathrm{HI}$ injury at P3. A1-A5: Anterior section of the brain immunostained for MBP. (A1) Coronal section of brain at the level of the striatum (10×): a reduction of the cortical and myelinated areas in the ipsilateral hemisphere extending from the cingulum to the rhinal sulcus can be seen. At $100 \times(A 2)$ and $400 \times(A 3)$ magnifications, the alteration of the myelin pattern is noticed when compared with the left hemisphere (A4 and A5). B1-B5: Midsection of the brain immunostained for MBP. (B1) Coronal section of brain at the level of the dorsal hippocampus $(10 \times)$ : a reduction of the cortical and myelinated areas in the ipsilateral hemisphere extending from the cingulum to the rhinal sulcus can be seen. At $100 \times(B 2)$ and $400 \times(B 3)$ magnifications, the alteration of the myelin pattern is noticed when compared with the left hemisphere (B4 and B5). C1-C3: Posterior section of the brain immunostained for MBP. (C1) Coronal section of brain at the level of the ventral hippocampus (10×): a reduction of the cortical and myelinated areas in the ipsilateral hemisphere extending from the cingulum to the rhinal sulcus can be seen. At $100 \times$ magnification $(C 2)$, the alteration of the myelin pattern is noticed when compared with the left hemisphere $(C 3)$.

injury in the $\mathrm{P} 3$ rat. The size of the hippocampus, striatum, thalamus, and corpus callosum were not significantly altered. The ipsilateral cortex had marked alterations in the organization of the myelinated fibers in association with persistent gliosis. Controls and ligated-only animals showed no damage confirming that only the combination of hypoxia and ligation was required for this lesion.

The effect of neural immaturity on the pattern of lesion after $\boldsymbol{H I}$. $\mathrm{HI}$ is an important cause of periventricular WM injury in the premature infant. These WM injuries occur at a time when there is rapid maturation of glia. This heightened susceptibility appears to occur against a backdrop of lowered vulnerability to cortical neuronal injury. In fetal sheep, the very preterm brain is relatively resistant to neuronal injury within the immature upper cortical plate neuronal layers, while sensitive to periventricular WM injury after HI injury (17). Similar selective sensitivity of WM in the immature rat and mouse brain have recently been reported after
HI injury (18-20) and after bilateral carotid occlusion in P5 rats (21). In this study, we have also observed a similar enhanced susceptibility to HI injury of the cortical WM in the very immature P3 rat compared with the more mature infant rat $(16,22)$. Thus, there is now compelling experimental evidence in distinct models indicating increased sensitivity of the developing cortical $\mathrm{WM}$ to $\mathrm{HI}$ injury in the immature brain.

Alteration in cortical gray matter. This model displayed patchy neuronal loss in the deeper layers (IV-VI) of the parietal cortex in association with altered myelination and disruption of the regular neuroarchitectural pattern. This selective loss of gray matter was associated with a trend toward a reduction in total gray matter volume that did not reach statistical significance. A similar pattern was observed in preterm fetal sheep (17). These patches were conical, with a columnar pattern, and limited to the deeper cortex (V-VI) rather than the full-thickness lesions seen in the widely used, more mature $\mathrm{P} 7$ rats after a unilateral $\mathrm{HI}$ injury (23). 


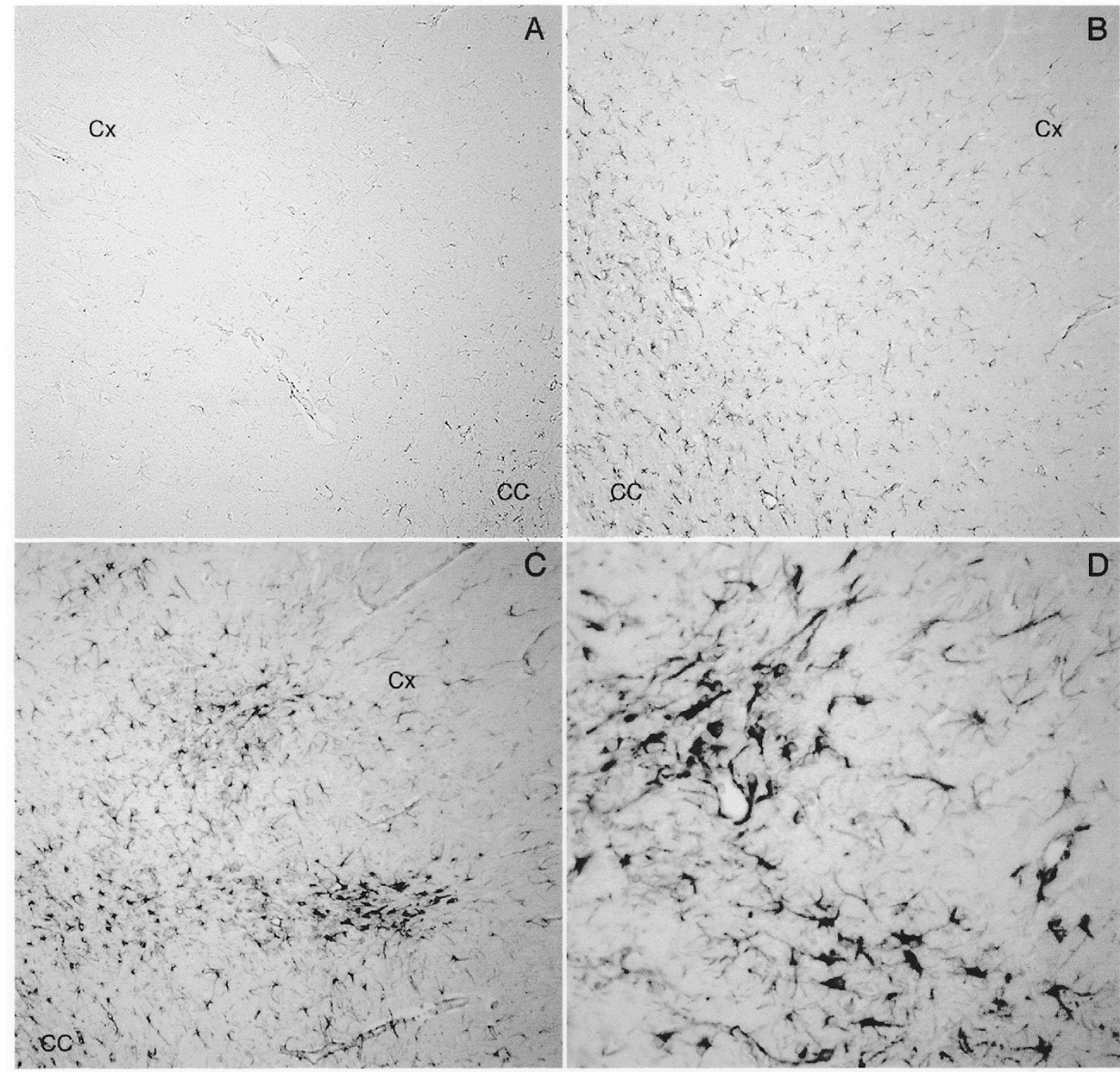

Figure 5. Pattern of gliosis in the deep parietal cortex at P21 after right HI injury at P3. Midsection of the brain immunostained for GFAP. (A) Contralateral parietal cortex of a mildly damaged brain $(16 \times)$ : rare GFAP-positive astrocytes were present. $(B)$ Ipsilateral parietal cortex of a mildly damaged brain $(16 \times)$ : note the diffuse pattern of GFAP-positive astrocytes localized in cortical layers IV-VI. (C) Ipsilateral parietal cortex of a severely damaged brain (16×): note the diffuse background with a denser columnar pattern of GFAP-positive astrocytes in cortical layers IV-VI. (D) High magnification (40 $\times$ ) of GFAP-positive hypertrophic astrocytes organized in a columnar pattern similar to the MBP immunostaining pattern. $C C$, corpus callosum; $C x$, cortex.

This columnar injury is thought to relate the distribution of immature penetrator vessels in the developing cortex (24). The pattern of lesion is similar to some of the findings of Towfighi $e t$ al. (22), despite their model of P2-3 HI being more severe than ours. This deep selective neuronal loss may reflect some of the loss of cortical gray matter volume seen in some infants who have been born prematurely (6).

Relevance of the nature and severity of injury. The neuropathological sequelae of the diffuse WM injury are hypomyelination, ventriculomegaly $(4,10)$, and marked loss of cortical cerebral WM volume with an absence of lesions in the basal ganglia and thalamus $(6,25)$. In this study, without any alteration to noncortical structures, overt cystic infarcts were not seen and the specific loss of WM appeared to be diffuse within the cortex. In contrast, the lesions in the widely used P7 unilateral $\mathrm{HI}$ rat model manifest as a full-thickness cortical infarction that develop cystic lesions over a period of weeks with damage in the hippocampus, striatum and, thalamus (23). Towfighi et al. $(22,26)$ carried out a number of studies using a number of age bands, including P2-3 rats. The exposure of $\mathrm{P} 2-3$ rats to a 60-min hypoxia with $5 \% \mathrm{O}_{2}$ resulted in 50\% mortality, with some surviving animals showing a columnar distribution of lesions that in some were fused to form infarcts. In both the P7 and P2-3 rats, the lesions were severe in nature and did not reflect the subtle alterations in neuropathology that would be more clinically relevant to the human premature infant. There was no data published on changes to subsequent myelin development in the P2-3 rat studies. The differences in reported outcome between our model and that published by Towfighi et al. presumably results from differences in the level of hypoxia and environmental temperature. Recently, Liu et al. (20) showed an alteration of MBP after a mild-to-severe $\mathrm{HI}$ injury in the P7 rat. An early loss (P12) of MBP immunostaining in the CC and striatum with a reduction of hemispheric size was reported. A restoration of the MBP staining in the mildly injured animals was 
present at P21 and indicated either functional recovery of injured oligodendrocytes or proliferation and maturation of oligodendroglial precursors. In our P3 moderate HI model, the persistent alterations in cortical WM with ongoing gliosis seen at P21 is thought to reflect the greater vulnerability to injury of oligodendrocytes progenitors that are present at this age (27). It could also reflect axonal damage leading to altered myelination. Nevertheless, the cortical myelination and development deficits seen in our model appear relevant to some aspect of diffuse WM injury in the preterm. The mechanisms of altered cortical development seen in this study need further investigations to define which of the neuroaxonal unit or the oligodendrocytes progenitors are the primary target.

Alteration in deep cortical structure and myelination is associated with persistent gliosis. In the deep parietal cortex, the altered pattern of myelinated fibers suggests that the developing fiber tracts skirted areas of damage as development progressed. This is similar to the disruption of fiber tracks in the central WM when observed with diffusion tensor magnetic resonance imaging in preterm infant with PVL (7). During and after the stage of neural development studied, there is further axonal outgrowth from the developing cortical plate neurons (13). At P3, there is a transient population of subplate neurons that mature early and act as guideposts for these developing axons $(14,28)$. These mature neurons may be highly vulnerable to injury in the immature brain (29). Similar disruption of axonal outgrowth of the pyramidal neurons has been observed in Golgi-stained neuropathological samples from infants who have survived the preterm period (30). A significant complication of WM damage is the localized disruption of axon fibers resulting in the loss of inputs and outputs to and from the overlying areas of gray mater (31). The clinical correlate of even a minimal amount of such WM injury could be cerebral palsy, dyslexia, epilepsy, audiovisual deficits, and learning deficits. In addition, a persistent gliosis $18 \mathrm{~d}$ after the acute HI injury was present in a similar pattern as seen in animal models $(32,33)$ and in preterm infant with PVL $(34,35)$. Together, the gliotic scars with developmental and myelination alterations certainly contribute to neurodevelopmental deficits. However, it is not clear to what extent these functional deficits or the alterations in cortical structures result from the lesion or from alterations in subsequent cerebral development.

In conclusion, we have adapted the unilateral HI injury approach to the P3 rat. A moderate focal injury induced at P3 leads to specific subsequent alterations of myelination and deep cortical organization at P21. This developmental disruption with a specific loss of cortical volume, altered myelination, persistent gliosis, and axonal outgrowth appears similar to some of the findings in the human premature infant with PVL. This model may allow delineation of the nature, timing, and mechanisms of injury.

\section{REFERENCES}

1. Volpe JJ 1997 Overview: perinatal and neonatal brain injury. Ment Retard Dev Disabil Res Rev 3:1-2

2. Dammann O, Leviton A 1997 The role of perinatal brain damage in developmental disabilities: an epidemiologic perspective. Ment Retard Dev Disabil Res Rev 3:13-21
3. Volpe JJ 1997 Brain injury in the premature infant: neuropathology, clinical aspects and pathogenesis. Ment Retard Dev Disabil Res Rev 3:1-12

4. Leviton A, Gilles F 1996 Ventriculomegaly, delayed myelination, white matter hypoplasia, and "periventricular" leukomalacia: how are they related? Pediatr Neurol 15:127-136

5. Inder TE, Huppi PS 2000 In vivo studies of brain development by magnetic resonance techniques. Ment Retard Dev Disabil Res Rev 6:59-67

6. Inder TE, Huppi PS, Warfield S, Kikinis R, Zientara GP, Barnes PD, Jolesz F, Volpe JJ 1999 Periventricular white matter injury in the premature infant is followed by reduced cerebral cortical gray matter volume at term. Ann Neurol 46:755-760

7. Huppi PS, Murphy B, Maier SE, Zientara GP, Inder TE, Barnes PD, Kikinis R, Jolesz FA, Volpe JJ 2001 Microstructural brain development after perinatal cerebral white matter injury assessed by diffusion tensor magnetic resonance imaging. Pediatrics 107:455-460

8. Dammann O, Hagberg H, Leviton A 2001 Is periventricular leukomalacia an axonopathy as well as an oligopathy? Pediatr Res 49:453-457

9. Dammann O, Leviton A 1997 Maternal intrauterine infection, cytokines, and brain damage in the preterm newborn. Pediatr Res 42:1-8

10. Volpe JJ 2001 Neurobiology of periventricular leukomalacia in the premature infant Pediatr Res 50:553-562

11. Dobbing J, Sands J 1979 Comparative aspects of the brain growth spurt. Early Hum Dev 3:79-83

12. Clancy B, Darlington RB, Finlay BL 2001 Translating developmental time across mammalian species. Neuroscience 105:7-17

13. Dani JW, Armstrong DM, Benowitz LI 1991 Mapping the development of the rat brain by GAP-43 immunocytochemistry. Neuroscience 40:277-287

14. Honig LS, Herrmann K, Shatz CJ 1996 Developmental changes revealed by immunohistochemical markers in human cerebral cortex. Cereb Cortex 6:794-806

15. Back SA, Luo NL, Borenstein NS, Levine JM, Volpe JJ, Kinney HC 2001 Late oligodendrocyte progenitors coincide with the developmental window of vulnerability for human perinatal white matter injury. J Neurosci 21:1302-1312

16. Sirimanne ES, Guan J, Williams CE, Gluckman PD 1994 Two models for determin ing the mechanisms of damage and repair after hypoxic-ischaemic injury in the developing rat brain. J Neurosci Methods 55:7-14

17. Reddy K, Mallard CE, Guan J, Marks KA, Bennet L, Gunning MI, Gunn AJ, Gluckman PD, Williams CE 1998 Maturational change in the cortical response to hypoperfusion injury in the fetal sheep. Pediatr Res 43:674-682

18. Follett PL, Rosenberg PA, Volpe JJ, Jensen FE 2000 NBQX attenuates excitotoxic injury in developing white matter. J Neurosci 20:9235-9241

19. Skoff RP, Bessert DA, Barks JD, Song D, Cerghet M, Silverstein FS 2001 Hypoxicischemic injury results in acute disruption of myelin gene expression and death of oligodendroglial precursors in neonatal mice. Int J Dev Neurosci 19:197-208

20. Liu Y, Silverstein FS, Skoff R, Barks JD 2002 Hypoxic-ischemic oligodendroglial injury in neonatal rat brain. Pediatr Res 51:25-33

21. Uehara H, Yoshioka H, Kawase S, Nagai H, Ohmae T, Hasegawa K, Sawada T 1999 A new model of white matter injury in neonatal rats with bilateral carotid artery occlusion. Brain Res 837:213-220

22. Towfighi J, Mauger D, Vannucci RC, Vannucci SJ 1997 Influence of age on the cerebral lesions in an immature rat model of cerebral hypoxia-ischemia: a light microscopic study. Brain Res Dev Brain Res 100:149-160

23. Rice JEd, Vannucci RC, Brierley JB 1981 The influence of immaturity on hypoxicischemic brain damage in the rat. Ann Neurol 9:131-141

24. Rorke LB 1992 Anatomical features of the developing brain implicated in pathogenesis of hypoxic-ischemic injury. Brain Pathol 2:211-221

25. Fedrizzi E, Inverno M, Bruzzone MG, Botteon G, Saletti V, Farinotti M 1996 MRI features of cerebral lesions and cognitive functions in preterm spastic diplegic children. Pediatr Neurol 15:207-212

26. Towfighi J, Mauger D 1998 Temporal evolution of neuronal changes in cerebral hypoxia-ischemia in developing rats: a quantitative light microscopic study. Brain Res Dev Brain Res 109:169-177

27. Back SA, Han BH, Luo NL, Chricton CA, Xanthoudakis S, Tam J, Arvin KL, Holtzman DM 2002 Selective vulnerability of late oligodendrocyte progenitors to hypoxia-ischemia. J Neurosci 22:455-463

28. McConnell SK, Ghosh A, Shatz CJ 1989 Subplate neurons pioneer the first axon pathway from the cerebral cortex. Science 245:978-982

29. Volpe JJ 1996 Subplate neurons-missing link in brain injury of the premature infant? Pediatrics 97:112-113

30. Marin-Padilla M 1997 Developmental neuropathology and impact of perinatal brain damage. II: white matter lesions of the neocortex. J Neuropathol Exp Neurol 56:219-235

31. Kostovic I, Lukinovic N, Judas M, Bogdanovic N, Mrzljak L, Zecevic N, Kubat M 1989 Structural basis of the developmental plasticity in the human cerebral cortex: the role of the transient subplate zone. Metab Brain Dis 4:17-23

32. Tanaka H, Araki M, Masuzawa T 1992 Reaction of astrocytes in the gerbil hippocampus following transient ischemia: immunohistochemical observations with antibodies against glial fibrillary acidic protein, glutamine synthetase, and S-100 protein. Exp Neurol 116:264-274

33. Burtrum D, Silverstein FS 1994 Hypoxic-ischemic brain injury stimulates glial fibrillary acidic protein mRNA and protein expression in neonatal rats. Exp Neurol 126:112-118

34. Hirayama A, Okoshi Y, Hachiya Y, Ozawa Y, Ito M, Kida Y, Imai Y, Kohsaka S, Takashima S 2001 Early immunohistochemical detection of axonal damage and glial activation in extremely immature brains with periventricular leukomalacia. Clin Neuropathol 20:87-91

35. Deguchi K, Oguchi K, Takashima S 1997 Characteristic neuropathology of leukomalacia in extremely low birth weight infants. Pediatr Neurol 16:296-300 